\title{
Hyperoside Suppresses Osteoclasts Differentiation and Function through Down-Regulating TRAF6/p38 MAPK Signaling Pathway
}

Jun Zhu

Guizhou University

Min Zhang

Guizhou University

Xiong-Li Liu

guizhou university

Zhi-Gang Yin

guizhou university

Xiao-Xue Han

guizhou university

Hui-Juan Wang ( $\nabla$ hjwang1@gzu.edu.cn )

Guizhou University https://orcid.org/0000-0003-0641-4848

Ying zhou

Guizhou University Of Traditional Chinese Medicine

Research

Keywords: Hyperoside, Osteoclast, Differentiation, Bone resorption, TRAF6/p38 MAPK

Posted Date: June 30th, 2021

DOI: https://doi.org/10.21203/rs.3.rs-655369/v1

License: (c) (i) This work is licensed under a Creative Commons Attribution 4.0 International License.

Read Full License 


\title{
Hyperoside suppresses osteoclasts differentiation and function through down-regulating TRAF6/p38 MAPK signaling pathway
}

\author{
Jun Zhu ${ }^{1,2}$, Min Zhang ${ }^{2}$, Xiong-Li Liu², Zhi-Gang Yin ${ }^{1,2}$, Xiao-Xue Han², Hui-Juan Wang ${ }^{2 *} \&$ Ying Zhou ${ }^{2,3^{*}}$
}

\begin{abstract}
Background: Osteoporosis (OP) is a systemic metabolic bone disease that the bone resorption exceeds the bone formation, resulting in reduced bone mass, degeneration of the microstructure of bone tissue, and then increased bone fragility and fracture risk. Hyperoside (HP), as a natural product, can promote proliferation and differentiation of osteoblasts and presents a protective effect on ovariectomized (OVX) mice. However, the inhibitory effect of HP on osteoclasts (OCs) and the potential mechanism remains to be elucidated.
\end{abstract}

Methods: In this study, RAW264.7 cells were used to generate OCs induced by RANKL, and HP was applied to the cell model. The effect of HP on OCs differentiation by TRAP-positive cell counting and TRAP activity test; Bone resorption assay and actin ring formation assay were used to verify the effect of HP on OCs function; The expression levels of osteoclast-specific genes and proteins were proved by RT-PCR and Western blotting.

Results: HP significantly suppressed RANKL-induced OCs differentiation, function and the mRNAs expression levels of the osteoclast-related genes. Western Blotting results demonstrated that HP reduced the expression level of TNF receptor-associated factor 6 (TRAF6) and inhibited the p38 MAPK signing pathway by reducing the phosphorylation level of p38, which subsequently down-regulated the expression level of c-fos and NFATc1, ultimately, led to a decrease of the osteoclast-specific proteins CTSK and TRAP. In addition, TRAP-positive cell counting and TRAP activity test showed that HP could inhibit the differentiation of OCs; Bone resorption assay indicated that HP could restrain the OCs' bone resorptive activity. Actin ring formation assay showed HP can cause the shrinkage of osteoclasts and disruption of actin ring structure.

Conclusion: These results revealed that HP had an inhibitory effect on OCs by down-regulating TRAF6/p38 MAPK signaling pathway. Therefore, HP could be a promising natural compound for lytic bone diseases.

\section{Keywords}

Hyperoside, Osteoclast, Differentiation, Bone resorption, TRAF6/p38 MAPK

\section{Introduction}

Osteoporosis (OP), a disease of bone metabolism, is characterized by the promoted bone resorption by osteoclasts (OCs) and the insufficient of osteoblasts formation, resulting in loss of bone mass, deterioration of bone microstructure and increased risk of fracture. Under normal physiological conditions, osteoclast-mediated bone resorption and osteoblast-mediated bone formation are maintained in a balance state to keep the homeostasis of the skeletal system. Hence, the imbalance will lead to the occurrence of bone disease [1]. Overabsorption of OCs can lead to several lytic bone diseases, such as postmenopausal osteoporosis, inflammatory arthritis, and neoplastic bone metastasis, therefore, the strengthening of OCs activity is the main cause of most adults' bone diseases [2]. The World Health Organization reports [3] that more than 1.02 billion people are suffering from OP worldwide, and the number is expected to rise to 1.36 billion by 2030 , so the prevention and treatment of OP is vital to the improvement of citizens' health.

OPG/RANKL/RANK signaling pathway is a relatively mature theory of OCs differentiation at present [4]. Osteoprotegerin (OPG), it can act as a pseudo-receptor of RANKL in the process of bone transformation. After binding to RANKL, OPG can block the RANKL/RANK signaling pathway, thus inhibiting the differentiation and maturation of OCs [5]. When RANKL is combined with RANK, RANK initiates the transduction of intracellular signaling by recruiting docking proteins such as tumor necrosis factor receptor-related factors (TRAFs) [6]. TRAF6 is critical for OCs differentiation, then, the mitogen-activated protein kinase (MAPK) signaling pathway, NF-kB signaling pathway and anti-apoptotic serine/threonine kinase (Akt/PKB) signaling pathway are activated [7]. The MAPK signaling pathway mainly includes three signaling pathways: c-Jun N-terminal kinase (JNK), extracellular signal-regulated kinase (ERK) and p38. The p38 signaling pathway can up-regulate the expression of c-fos and promote its 
combination with nuclear factor of activated T-cell 1 (NFATc1), which is one of the major downstream targets of RANK signaling [8]. NFATc1 activates and induces the expression of OC specific genes through self-amplification and interaction with other transcription factors, which encodes proteins related to OCs differentiation, fusion and function [9], such as the tartrate-resistant acid phosphatase (TRAP), cathepsin K (CTSK) and dendritic cell-specific transmembrane protein (DC-STAMP). In this article, the TRAF6/p38 MAPK signaling pathway was mainly studied.

At present, some drugs with anti-osteoporosis effect are widely used in clinic, such as calcitonin, bisphosphonates, vitamin D and hormones (estrogen and parathyroid hormone), but the side effects of these drugs can not be ignored. A number of chinese herbal medicines and their natural products have been found to exhibit favorable anti-osteoporosis properties with minor side effects, which are more advantageous than chemical drugs and hormonal drugs [10]. HP, a flavonol glycoside compound, is widely found in various plants, such as the fruits and whole plants of Hypericaceae, Campanulaceae and Ericaceae. It was found that HP could protect mice from OP caused by ovariectomy [11], reduce the apoptosis and autophagy rate of MC3T3-E1 [12], and attenuate inflammation in C57BL/6J mice with acute liver injury (ALI), and reduce the expression of TNF- $\alpha$ and IL-6 in lipopolysaccharide (LPS)-induced RAW264.7 cells [13]. But, the effect of HP on the differentiation and function of osteoclasts and its potential molecular mechanism have not been reported. In this article, we utilized the cell model of RAW264.7 induced by RANKL, combined with TRAP staining, determination of TRAP activity and bone resorption activity assay, to study the effect of HP on OCs differentiation and bone resorption activity, meanwhile, the molecular mechanism of HP on OCs was explored through TRAF6/p38 MAPK signaling pathway.

\section{Methods}

\section{Materials, reagents and antibodies}

Hyperoside, purity $>98 \%$, was purchased from Chengdu Pusi Biotechnology Co., L td (Chengdu, China). Estradiol, 3-(4,5-Dimethylthiazol-2-yl)-2,5-diphenyltetrazolium bromide (MTT) and TritanX-100 were purchased from Beijing Solarbio Biological Technology Co., Ltd (Beijing, China). Mouse recombinant RANKL protein was obtained from R\&D Systems (Minneapolis, MN, USA). Fetal Bovine Serum was obtained from Zhejiang Tianhang Biotechnology Co., Ltd (Zhejiang, China). LEUKDCYTE ACID PHO SPHATASE (TRAP) KIT and toluidine blue was purchased from Sigma System (USA). tartaric-resistant phosphatase (StrACP) test kit was obtained from Nanjing Jiancheng Institute of Bioengineering (Nanjing, China). The bone slices was purchased from Orthopedic Hospital of Lanzhou Military Region (Gansu, China). Phalloidin-Tetramethylrhodamine Conjugate was purchased from Shanghai Absin Company (Shanghai, China). BCA Total Protein Quantitative Kit was obtained from Beyotime (Shanghai, China). RT EasyTM and Real Time PCR Easy TM-SYBR Green I kit was purchased from FOREGENE (Chengdu, China). Total RNA Kit I (Omega BIO-TEK, USA). Rabbit anti-mouse $\beta$-actin, c-fos, NFATc1 primary antibodies were purchased from Cell Signaling Technology (Danvers, USA). Rabbit anti-mouse CTSK, TRAP, TRAF6 primary antibodies were obtained from Abcam (Cambridge, UK). Goat anti-rabbit secondary antibody was obtained from Shanghai Absin Company (Shanghai, China).

\section{Cell culture}

RAW264.7 cells were cultured in 10\% FBS medium (DMEM high glucose medium plus 10\% FBS, $100 \mathrm{ug} / \mathrm{ml}$ penicillin strepthromycin and $2 \mathrm{mM}$ glutamine). In this study, cells were incubated in $\mathrm{CO}_{2}$ incubator (Thermo Fisher Scientifie, USA) at constant high humidity, $37^{\circ} \mathrm{C}$, and $5 \% \mathrm{CO}_{2}$ atmosphere.

\section{Cytotoxicity assay}

The cells were seeded into 96-well plates, which were randomly divided into experimental groups and control group. In the experimental groups, different concentrations of $\mathrm{HP}(0.01,0.1,1,10,100$ and $1000 \mu \mathrm{M})$ were added to the $10 \% \mathrm{FBS}$ medium. After $3 \mathrm{~d}$, MTT (5 mg/l, Solarbio, China) was added to all wells, the plates were incubated for $2 \mathrm{~h}$, and the absorbance value was measured at $490 \mathrm{~nm}$ using a multifunctional microplate reader (Thermo Fisher Scientifie, USA). Survival rate was calculated relative to the control group using the following formula: (experimental group OD - zeroing OD)/(control group OD -zeroing OD) $\times 100 \%$. 
RAW264.7 cells were divided into experimental groups: 10\% FBS medium contains different concentrations of HP (10, 25 and 50 $\mu \mathrm{M}$ ) and RANKL (30 ng/ml), the negative control: 10\% FBS medium contained RANKL (30 ng/ml) and the positive control: 10\% FBS medium contained estradiol $\left(10^{-2} \mu \mathrm{M}\right)$ and RANKL $(30 \mathrm{ng} / \mathrm{ml})$.

\section{TRAP staining}

RAW264.7 cells were inoculated with or without $\mathrm{HP}(10,25$ and $50 \mu \mathrm{M})$ or estradiol $\left(10^{-2} \mu \mathrm{M}\right)$ in an osteoclast-inducing media for $5 \mathrm{~d}$. Cultured cells were fixed with $10 \%$ formaldehyde, and stained according to LEUKDCYTE ACID PHOSPHATASE (TRAP) KIT (Sigma System, USA). The TRAP-positive cells (usually more than 3 nuclei) were counted under an inverted fluorescence microscope (Leica-microsystems, Germany).

\section{Determination of TRAP activity}

RAW264.7 cells were seeded in 48-well plates, cultured with or without HP $(10,25$ and $50 \mu \mathrm{M})$ or estradiol $\left(10^{-2} \mu \mathrm{M}\right)$ in an osteoclast-inducing media for $5 \mathrm{~d}$. The cells were rinsed with PBS after cell induction. 1\% TritanX-100 (Solarbio, China) was added to the cells in an incubator at $37^{\circ} \mathrm{C}$ for $5 \mathrm{~min}$. The obtained lysis solution was detected in accordance with the tartaric-resistant phosphatase (StrACP) test kit (Nanjing Jiancheng Institute of Bioengineering, China).

\section{Bone resorption activity assay}

The bone slices (Orthopedic Hospital of Lanzhou Military Region, China) were cut into appropriate sizes $(0.5 \mathrm{~cm} \times 0.5 \mathrm{~cm})$ and placed into a 48-well plate. RAW264.7 cells were cultured with or without HP $(10,25$ and $50 \mu \mathrm{M})$ or estradiol $\left(10^{-2} \mu \mathrm{M}\right)$ in an osteoclast-inducing media for $7 \mathrm{~d}$. The bone slices were firstly fixed with $10 \%$ formaldehyde, then, sonicated with $0.25 \mathrm{M}$ ammonia water. $80 \%, 85 \%, 90 \%$ and $100 \%$ ethanol were used for gradient elution, and finally the bone slices was stained with $0.1 \%$ toluidine blue (Sigma system, USA). The bone slices were observed and photographed by a microscope, and the area of the bone resorption pits was calculated.

\section{Actin ring formation assay}

RAW264.7 cells were seeded in 96-well plates, inoculated with or without $\operatorname{HP}(10,25$ and $50 \mu \mathrm{M})$ or estradiol $\left(10^{-2} \mu \mathrm{M}\right)$ in an osteoclast-inducing media for $5 \mathrm{~d}$. Cells were washed with PBS, fixed in $10 \%$ formaldehyde in PBS for 30 minutes and added $0.1 \%$ Triton X-100 in PBS into fixed cells for 5 minutes to increase permeability. Rinse the fixed cells 3 times, then incubated with Phalloidin-Tetramethylrhodamine Conjugate $(1 \mu \mathrm{g} / \mathrm{ml})$ for $30 \mathrm{~min}$. F-actin stained with Phalloidin-Tetramethylrhodamine Conjugate was photographed using a fluorescence microscope at a wavelength of $546 \mathrm{~nm}$.

\section{Real-time PCR analysis}

RAW264.7 cells were cultured in 6-well plates with or without HP $(10,25$ and $50 \mu \mathrm{M})$ or estradiol $\left(10^{-2} \mu \mathrm{M}\right)$ in an osteoclast-inducing media for $5 \mathrm{~d}$. The induced cells were subjected to RNA extraction according to Total RNA Kit I (Omega BIO-TEK, USA). Reverse transcription conditions and PCR reaction conditions were in accordance with RT EasyTM (FOREGENE, China) and Real Time PCR Easy TM-SYBR Green I kit (FOREGENE, China) respectively. The PCR primers were designed as follows: TRAF6, 5'-AGTATGAGTGTCCCATCTGCT-3' and 5'-TTTACCGTCAGGGAAAGAAT-3'; c-fos, 5'-GGTTTCAACGCCGACTACGA-3' and 5'-AGCCCATGCTGGAGAAGGA-3'; NFATc1, 5'-GCCTCGAACCCTATCGAGT G-3' and 5'-TCCCGGTCAGTCTTTGCTTC-3'; CTSK, 5'-CATGCAGTGTTGGTGGTGGG-3' and 5'-CCGCAGGCGTTGTTC TTATT-3'; TRAP, 5'-ACGATGCCAGCGACAAGAGG-3' and 5'-TTCCAGCCAGCACATACCAG-3'; $\beta$-actin, 5'-CTGTCCC TGTATGCCTCT-3' and 5'-ATGTCACGCACGATTTCC-3'. Using $\beta$-actin as an internal reference, the mRNAs expression levels of osteoclast-related genes were calculated by the $2^{-\Delta \Delta c t}$ method [14].

\section{Western Blot analysis}

Total protein was extracted using RIPA lysis buffer (containing 1\% protease inhibitor). The total protein concentration was determined according to the BCA Total Protein Quantitative Kit (Beyotime, China). The sample proteins (20mg) were resolved on $8 \%$ sodium dodecyl sulfate polyacrylamide gel (SDS-PAGE), and then the protein was transferred to polyvinylidene difluoride membrane (Immobilon-P Transfer Membrane, Ireland). 5\% skimmed milk powder in TBS-Tween (25 mM Tris-HCl, $\mathrm{pH} 7.4 ; 150$ 
$\mathrm{mM} \mathrm{NaCl}$; and $0.1 \%$ Tween 20) was used for blocking for $1 \mathrm{~h}$. Incubating the primary antibody (rabbit anti-mouse) overnight at $4^{\circ} \mathrm{C}$, then, the secondary antibody (goat anti-rabbit) was used to incubate membranes for $1 \mathrm{~h}$. Protein signals were detected using the Immobilon Western HRP Substrate Peroxide Solution (Burlington, MA, USA) and the V3 Western Blotting System (Bio-Rad, USA). The band intensities of each protein was compared with $\beta$-actin, then, the ratio was compared with the negative control to obtain the relative quantification [15].

\section{Statistical analysis}

All experiments were conducted three times. The experimental data expressed as mean \pm standard deviation (SD) and compared by the two-tailed Student's t-test or one-way analysis of variance (ANOVA) with Tukey's multiple comparison post-hoc test. $\mathrm{P}<0.05$ was considered statistically significant.

\section{Results}

\section{HP inhibited the osteoclastogenesis induced by RANKL}

Before studying the effect of HP on the differentiation of RAW264.7 cells into OCs, we observed the effect of HP on the survival rate of RAW264.7 cells. The survival rate of the cells in $1000 \mu \mathrm{M}$ decreased significantly (89\%), while 100, 10, 1, 0.1 , and $0.01 \mu \mathrm{M}$ gradually increased, and the survival rate was above 95\% (Fig. 1B). Hence, HP below $100 \mu \mathrm{M}$ can be considered less toxic to RAW264.7 cells [16]. In our study, 10, 25 and $50 \mu \mathrm{M}$ HP were chosen to study the effect on the osteoclastogenesis of RAW264.7 cells. RAW264.7 cells were continuously induced by RANKL for 5 d. After staining, it was found that some of RAW264.7 cells differentiated into large, multinucleated cells (usually more than 3), which were regarded as TRAP positive OCs [17]. As can be seen in Fig. 1A, OCs in the negative control were most strongly generated, with a large number and volume. With the increase of HP concentration, the number of TRAP positive cells decreased. The statistical results of TRAP positive cells were shown in Fig. 1C. In addition, we found that HP suppressed the TRAP activity of RANKL-induced OCs in a dose-dependent manner, showing in Fig. 1D. Compared with the negative control, HP significantly inhibited osteoclastogenesis, and $50 \mu \mathrm{M}$ HP exhibited the best inhibitory effect.

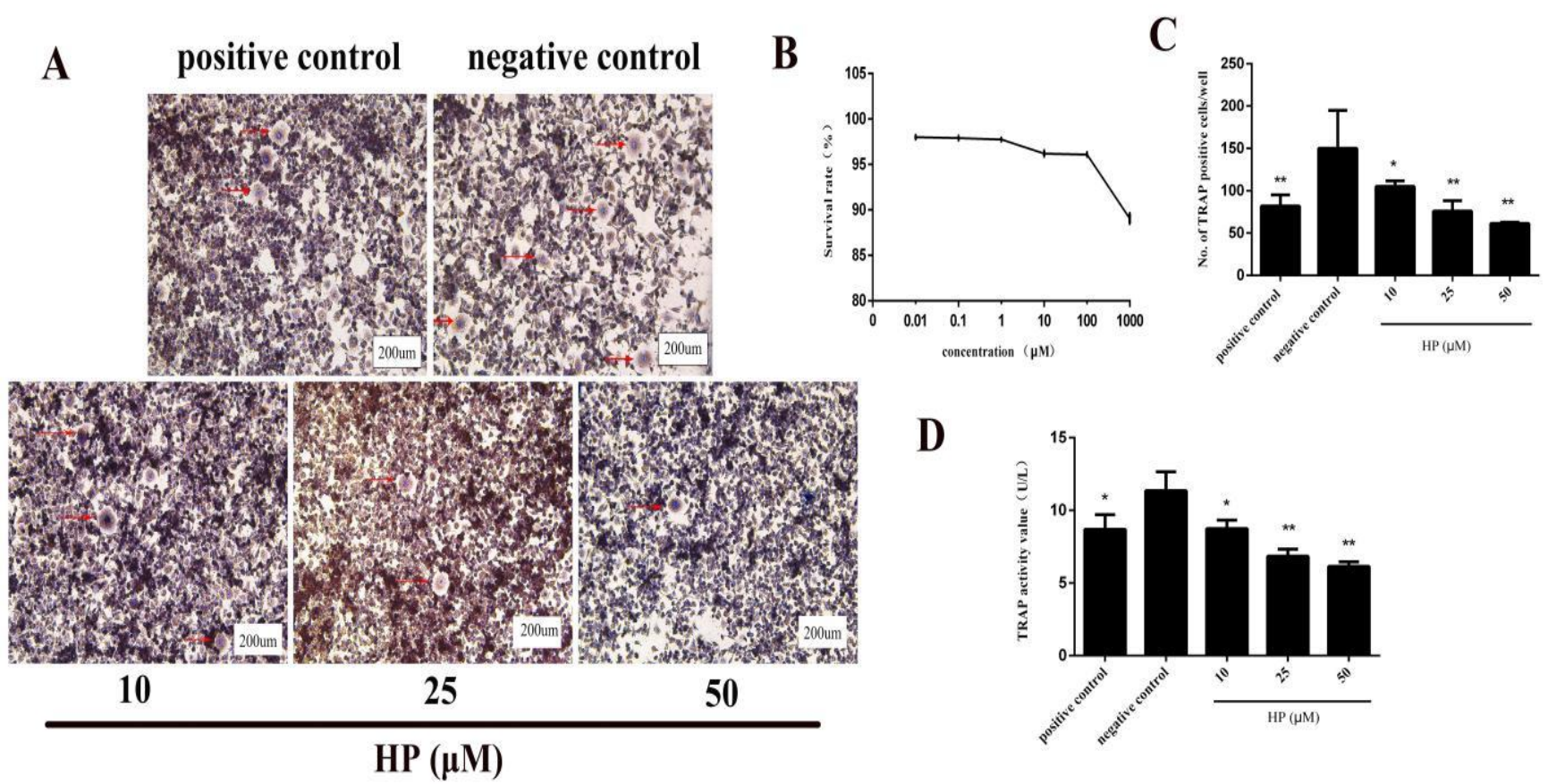

Fig. 1. HP inhibited the osteoclastogenesis induced by RANKL. (A) RAW264.7 cells were inoculated with or without HP (10, 25 and $50 \mu \mathrm{M})$ or estradiol $\left(10^{-2} \mu \mathrm{M}\right)$ in an osteoclast-inducing media for $5 \mathrm{~d}$. The cells were stained to count the number of TRAP positive cells. (B) RAW264.7 cells were inoculated with different concentrations of HP for $3 \mathrm{~d}$, then the survival rate was measured by the MTT assay. (C) The number of TRAP positive cells. (D) The TRAP activity was detected after cells induction. All values represent mean $\pm \mathrm{SD} . \mathrm{N}=3 . * \mathrm{P}<0.05$ and $* * \mathrm{P}<0.01$ versus the negative control. 
Under the induction of RANKL, RAW264.7 cells differentiated into OCs. OCs adsorbed on the bone slices to perform the function of bone resorption and the resorption pits on the bone surface were obviously observed through the staining of toluidine blue. OCs are the only cells in vivo to perform the function of bone resorption, and the area of bone pits can reflect and evaluate the bone resorption capacity of OCs [18]. It could be seen from Fig. 2A that the bone resorption area of the negative control was the largest. With the increase of HP concentration, the bone resorption area gradually decreased. The statistical results of the area of bone resorption pits were shown in Fig. 2B. The result indicated that HP had an inhibitory effect on the bone resorption function of OCs.

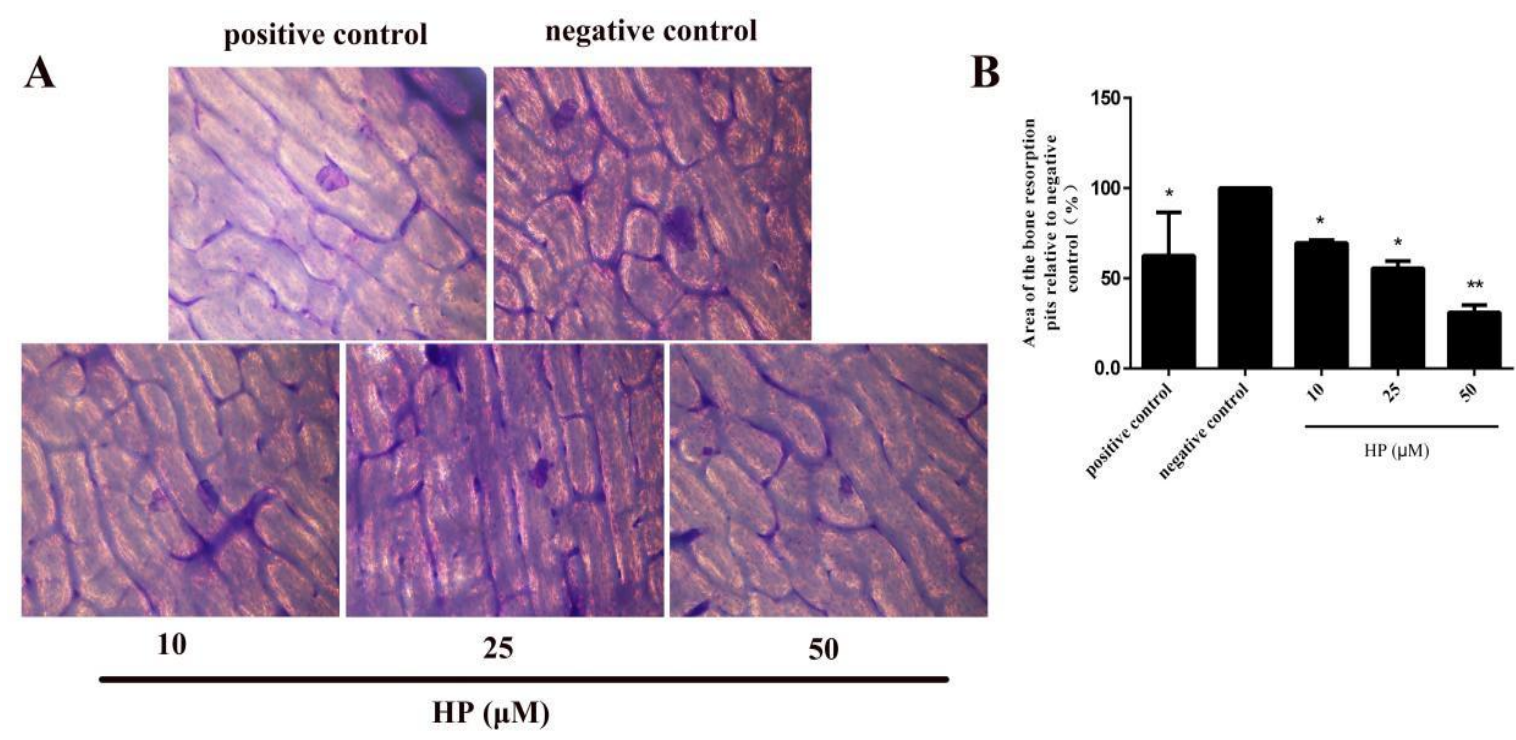

Fig. 2. HP attenuated the OCs' bone resorption function. (A) RAW264.7 cells were cultured with or without HP (10, 25 and $50 \mu \mathrm{M}$ ) or estradiol $\left(10^{-2} \mu \mathrm{M}\right)$ in an osteoclast-inducing media for $7 \mathrm{~d}$. The bone slices were stained with $0.1 \%$ toluidine blue. (B) Area of the bone resorption pits relative to the negative control. All values represent mean $\pm \mathrm{SD}$. $\mathrm{N}=3$. $* \mathrm{P}<0.05$ and $* * \mathrm{P}<0.01$ versus the negative control.

\section{Inhibitory effect of HP on actin ring structure of mature osteoclasts.}

The bone resorption function of osteoclasts depends on dynamic regulation of the actin cytoskeleton. Actin ring structure is a characteristic cytoskeletal feature of functional osteoclasts. Therefore, we next examined whether HP affects actin ring structure of mature osteoclasts. In mature osteoclasts on tissue culture plates, F-actin was arranged into a ring-like structure (actin ring) at the cell periphery [19]. In Figure 3A and B, the treatment of mature osteoclasts with HP caused both shrinkage of osteoclasts and disruption of actin ring structure in a dose-dependent manner, and the number of intact actin rings gradually decreased with the increase of HP concentration.

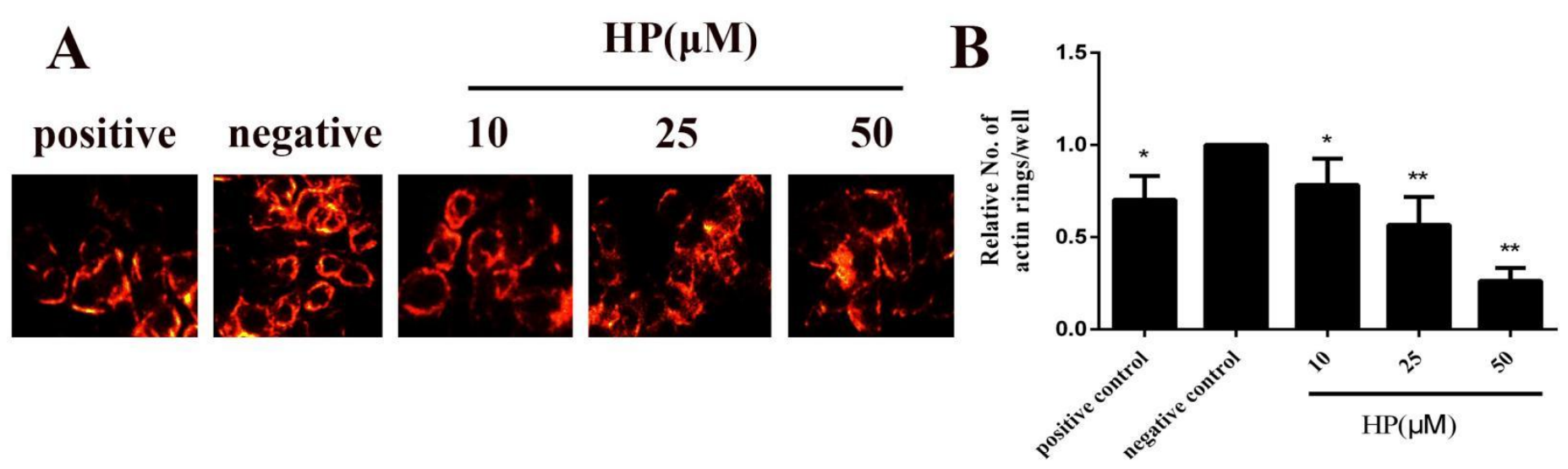

Fig. 3. Inhibitory effect of HP on actin ring structure of mature osteoclasts. RAW264.7 cells were cultured with or without HP $(10,25$ and $50 \mu \mathrm{M})$ or estradiol $\left(10^{-2} \mu \mathrm{M}\right)$ in an osteoclast-inducing media for $5 \mathrm{~d}$. Osteoclasts were fixed, and F-actin was visualized by fluorescence microscope with Phalloidin-Tetramethylrhodamine Conjugate staining. All values represent mean $\pm \mathrm{SD} . \mathrm{N}=3 . * \mathrm{P}<0.05$ and $* * \mathrm{P}<0.01$ versus the negative control. 


\section{HP inhibited the mRNAs expression levels of osteoclast-related genes}

In order to further demonstrate the role of HP in the differentiation of OCs, we studied the mRNA expression levels of osteoclast-related genes by real-time PCR. RAW264.7 cells induced by RANKL were treated with different concentration of HP. As shown in Fig. 3A, compared with the negative control, the mRNAs expression levels of TRAF6, c-fos, NFATc1, CTSK, and TRAP exhibited a downward trend. TRAF6 can initiate the p38 MAPK signaling pathway, and up-regulate the expression levels of c-fos and NFATc1, ultimately leading to the fusion and maturation of OCs. TRAP and CTSK are related to the executive function of OCs. The results explained that HP inhibited OCs differentiation by regulating the mRNAs expression levels of these genes.

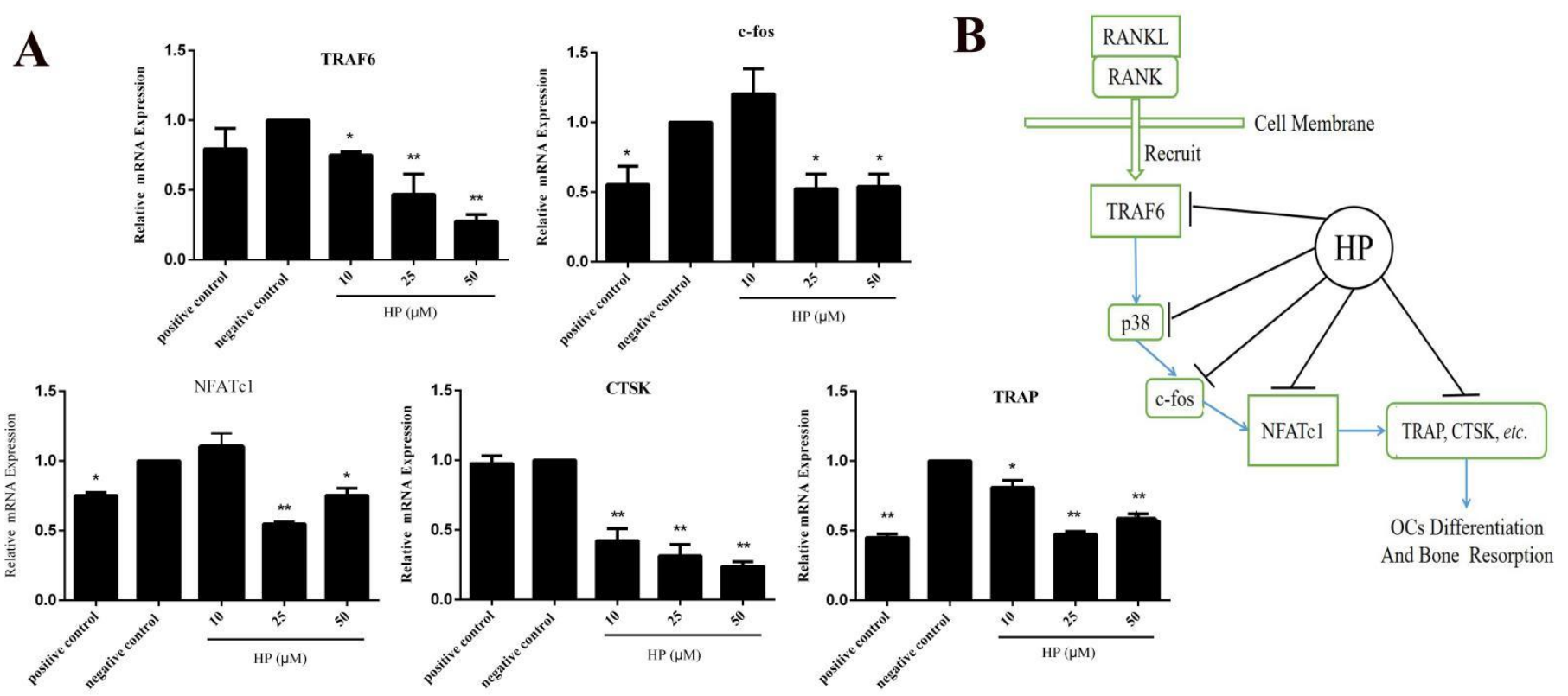

Fig. 4. HP suppressed the mRNAs expression levels of osteoclast-related pathway genes. (A) RAW264.7 cells were cultured with or without HP $(10,25$ and $50 \mu \mathrm{M})$ or estradiol $\left(10^{-2} \mu \mathrm{M}\right)$ in an osteoclast-inducing media for 5 d. HP down-regulated the mRNAs expression levels of TRAF6, c-fos, NFATc1, CTSK and TRAP. (B) Schematic diagram of HP inhibiting TRAF6/p38 MAPK signaling pathway. All values represent mean $\pm \mathrm{SD} . \mathrm{N}=3 . * \mathrm{P}<0.05$ and $* * \mathrm{P}<0.01$ versus the negative control.

\section{HP inhibited the proteins expression levels of osteoclast-related signaling pathway}

To examine the molecular mechanism of the anti-osteoclastogenic action of HP, RAW264.7 cells induced by RANKL were treated with different concentration of HP. Western Blotting results were shown in Fig. 4A and Fig. 4B. Compared with the negative control, the proteins expression levels of TRAF6, c-fos, NFATc1, TRAP, CTSK and the phosphorylation level of p38 MAPK decreased. These proteins are related to OCs differentiation in the upstream and downstream of the TRAF6/p38 MAPK signaling pathway. The results suggested that HP exerted an anti-osteoclastogenic effect by regulating the TRAF6/p38 MAPK signaling pathway. 
A
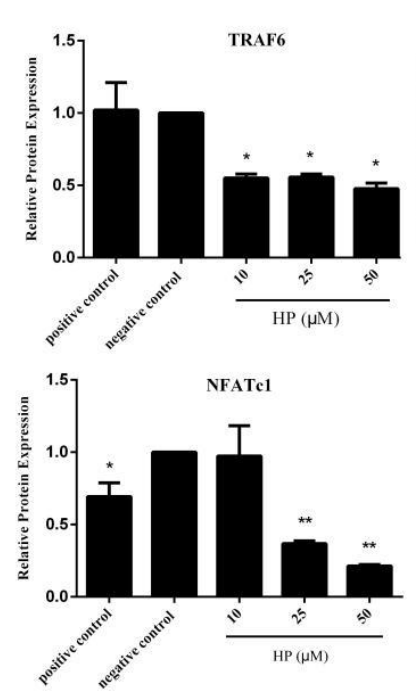
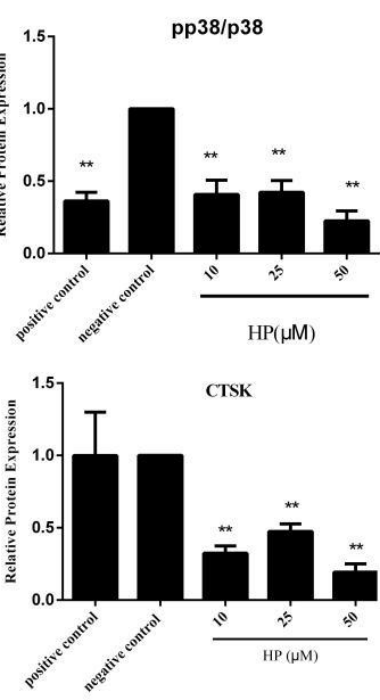

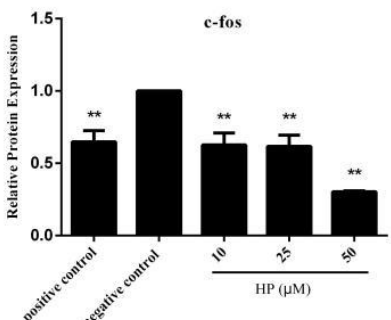

TRAP

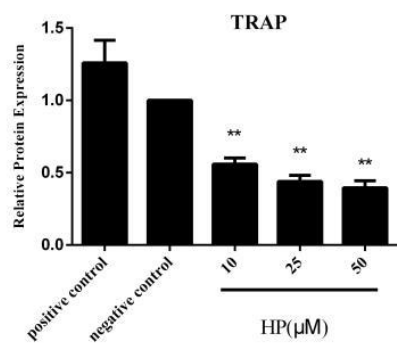

B

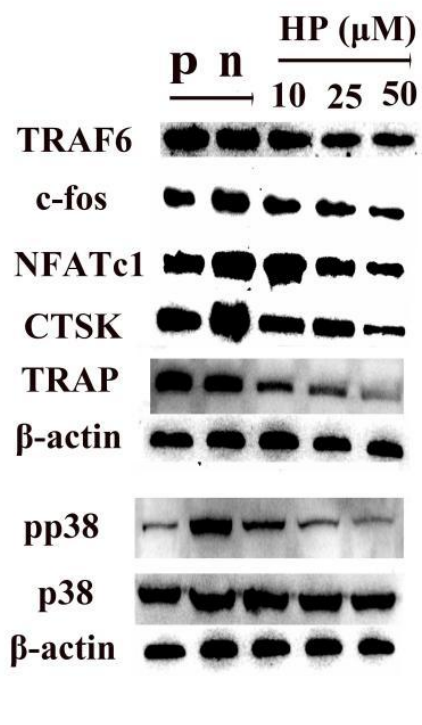

Fig. 5. HP inhibited the proteins expression levels of osteoclast-related signaling pathway. (A) RAW264.7 cells were cultured with or without HP $(10,25$ and $50 \mu \mathrm{M})$ or estradiol $\left(10^{-2} \mu \mathrm{M}\right)$ in an osteoclast-inducing media for $5 \mathrm{~d}$. The graphs showed the quantification of band intensities for TRAF6, pp38/p38, c-fos, NFATc1, TRAP and CTSK to $\beta$-actin. (B) The expression of TRAF6, pp38, p38, c-fos, NFATc1, Trap and CTSK proteins were analyzed by Western Blotting; p: the positive control; n: the negative control. All values represent mean $\pm \mathrm{SD} . \mathrm{N}=3 .{ }^{*} \mathrm{P}<0.05$ and $* * \mathrm{P}<0.01$ versus the negative control.

\section{Discussion}

HP, as a kind of flavonoids, is found in many chinese herbal medicines. In recent years, HP has also been studied in anti-osteoporosis. HP can protect MC3T3-E1 from oxidative stress via inhibiting the MAPK signaling pathway and oxidative damage of the cells [20], reduce the apoptosis and autophagy rate of MC3T3-E1. In addition, HP also has a good anti-osteoporosis performance in OVX mice through NF- $\kappa \mathrm{B}$ signaling pathway.

In this research, we studied the effect of HP on RANKL induced OCs from RAW264.7 cells, including TRAP-positive cell counting and TRAP activity test. TRAP, as a landmark phosphatase in osteoclasts, can reflect the inhibitory ability of HP on osteoclastogenesis. As shown in Fig. 1A and C, compared with the negative control, HP groups $(10,25$ and $50 \mu \mathrm{M})$ gradually decreased the number of TRAP positive cells. In Fig. 1D, TRAP activity in HP groups significantly decreased in a concentration dependent-manner. As shown in Fig. 2A and B, compared with the negative control, HP groups reduced the area of bone resorption pits significantly. The above results indicated that HP could suppress the differentiation and bone resorption capacity of OCs.

The assembly of F-actin to form an organized ring is necessary for osteoporotic function. Mature osteoclasts will form a fold boundary rich in F-actin structure on the bone surface, separating the absorption cavity from the extracellular space. The sealing area forms a large circular F-actin band and contains densely podosomes as an adhesion structure. In mature osteoclasts, the podosomes will dynamically arrange into a specialized near-membrane ring to migrate and absorb bone. These osteoclast-specific structures are essential for osteoclasts to perform their functions [21-22]. As shown in Fig. 3A and B, compared with the negative control, HP groups caused both the shrinkage of osteoclasts and disruption of actin ring structure, and the number of intact actin rings gradually decreased in a dose-dependent manner.

In the signaling pathway of RANKL/RANK/TRAF6, NFATc1 plays a crucial role. The function of NFATc1 depends on three stages: initiation, amplification and targeting [23]. The initiation and amplification of NFATc1 mainly rely on its upstream signal regulation, such as the combination of RANKL and RANK. TRAF6 mediates the up-expression of signal molecules such as NF-kB, c-Jun and p38 after its recruitment, and activates the downstream transcription process of NFATc1 [24]. It is reported [25] that the combination of c-fos, activated by p38, and NFATc1 can induce a increase expression level of NFATc1. NFATc1 affects the differentiation, fusion and function of OCs through the induction and expansion of target cell mRNA levels. While OC precursor cells fused and OCs perform functions, the proteins encoded by the downstream target gene of NFATc1 play an important role, such 
as TRAP, CTSK, DC-STAMP and tonoplast proton pump subunit (atp6v0d2) [26-27]. Among them, CTSK can denature bone collagen [28], TRAP can dephosphorylate the bone matrix phosphate osteopontin and the bone sialoglycoprotein [29-30].

Collectively, HP inhibited the expression level of TRAF6, and attenuated the phosphorylation of p38, so that the expression of c-fos and NFATc1 were down-regulated, and ultimately, the differentiation and bone resorption capacity of OCs were inhibited. As shown in Fig. 4 and Fig. 5, HP had a certain inhibitory effect on the expression levels of genes and proteins related to the TRAF6/p38 MAPK signaling pathway.

\section{Conclusions}

In summary, this study demonstrated that HP had an inhibitory effect on the differentiation and function of OCs in the cell model of RAW264.7 induced by RANKL, and the underlying molecular mechanism was associated with the inhibition of TRAF6/p38 MAPK signaling pathway. These results suggest that HP could be a promising natural compound for lytic bone diseases.

\section{List of abbreviations}

OP: Osteoporosis; HP: Hyperoside; OCs: osteoclasts; OVX: ovariectomized mice; TRAF6: TNF receptor-associated factor 6; OPG: Osteoprotegerin; MAPK: mitogen-activated protein kinase; NFATc1: nuclear factor of activated T-cell 1; TRAP: tartrate-resistant acid phosphatase; CTSK: cathepsin K; DC-STAMP: dendritic cell-specific transmembrane protein.

\section{Ethics approval and consent to participate}

Not applicable.

\section{Consent to publish}

Not applicable.

\section{Availability of data and materials}

The datasets used and analyzed during the current study are available from the corresponding author on reasonable request.

\section{Funding}

This research was financially supported by the National Key R\&D Program of China (2018YFC1708100); Guizhou Science and Technology Program (Qian Ke He Ji Chu ZK [2021] 520); Guizhou University Introduced Talent Research Project (Gui Da Ren Ji He Zi [2019] 29).

\section{Competing interests}

The authors have no conflicting interests.

\section{Acknowledgements}

Not applicable.

\section{Author details}

${ }^{1}$ Key laboratory of Plant Resource Conservation and Germplasm Innovation in Mountainous Region (Ministry of Education), Collaborative Innovation Center for Mountain Ecology \& Agro-Bioengineering (CICMEAB), Institute of Agro-bioengineering, College of Life Sciences, Guizhou University, Guiyang 550025, China; ${ }^{2}$ National \& Local Joint Engineering Research Center for the Exploition of Homology Resources of Southwest Medicine and Food, Guizhou University, Guiyang, 550025, China; ${ }^{3}$ College of pharmacy, Guizhou University of Traditional Chinese Medicine, Guiyang, 550025, China

\section{Authors' contributions}


Hui-Juan Wang and Ying Zhou conceived the study. Min Zhang, Xiong-Li Liu and Zhi-Gang Yin designed the study. Jun Zhu and Xiao-Xue Han performed the experiment assays. Jun Zhu wrote the manuscript. All authors read and approved the final manuscript.

\section{References}

1. Appelman-Dijkstra NM, Papapoulos SE. Modulating Bone Resorption and Bone Formation in Opposite Directions in the Treatment of Postmenopausal Osteoporosis [J]. Drugs, 2015, 75 (10): 1049-58.

2. Novack DV, Teitelbaum SL. The osteoclast: friend or foe? [J]. Annu Rev Pathol, 2008, 3: 457-84.

3. Stevens JA, Rudd RA. The impact of decreasing U.S. hip fracture rates on future hip fracture estimates [J]. Osteoporos Int, 2013, 24 (10): 2725-8.

4. Baud'huin M, et al. Key roles of the OPG-RANK-RANKL system in bone oncology [J]. Expert Rev Anticancer Ther, 2007, 7 (2): 22 1-32.

5. Khosla S. Minireview: the OPG/RANKL/RANK system [J]. Endocrinology, 2001, 142 (12): 5050-5.

6. Boyce BF, Xing L. Biology of RANK, RANKL, and osteoprotegerin [J]. Arthritis Res Ther, 2007,9 Suppl 1 (Suppl 1): S1.

7. Kim B, Lee KY, Park B. Icariin abrogates osteoclast formation through the regulation of the RANKL-mediated TRAF6/NF-אB/ERK signaling pathway in Raw264.7 cells [J]. Phytomedicine, 2018, 51: 181-190.

8. Itzstein C, Coxon FP, Rogers MJ. The regulation of osteoclast function and bone resorption by small GTPases [J]. Small GTPases, 2011, 2 (3): 117-130.

9. Kang JY, et al. The Role of Ca2+-NFATc1 Signaling and Its Modulation on Osteoclastogenesis [J]. Int J Mol Sci, 2020, 21 (10): 3646.

10. An J, et al. Natural products for treatment of osteoporosis: The effects and mechanisms on promoting osteoblast-mediated bone formation [J]. Life Sci, 2016 147: 46-58.

11. Chen Y, et al. Beneficial effects of hyperoside on bone metabolism in ovariectomized mice [J]. Biomed Pharmacother, 2018, 107: $1175-1182$.

12. Zhang Q, Zhang XF. Hyperoside decreases the apoptosis and autophagy rates of osteoblast MC3T3-E1 cells by regulating TNF-like weak inducer of apoptosis and the p38mitogen activated protein kinase pathway [J]. Mol Med Rep, 2019, 19 (1): 41-50.

13. Huang $\mathrm{C}$, et al. Hyperin attenuates inflammation by activating PPAR- $\gamma$ in mice with acute liver injury (ALI) and LPS-induced RAW264.7 cells [J]. Int Immunopharmacol, 2015, 29 (2): 440-447.

14. Singh C, Roy-Chowdhuri S. Quantitative Real-Time PCR: Recent Advances [J]. Methods Mol Biol, 2016, 1392: $161-76$.

15. Hirano S. Western blot analysis [J]. Methods Mol Biol, 2012, 926: 87-97.

16. Kumar P, Nagarajan A, Uchil P. Analysis of Cell Viability by the MTT Assay [J]. Cold Spring Harb Protoc, 2018, 2018 (6).

17. Lindunger A, et al. Histochemistry and biochemistry of tartrate-resistant acid phosphatase (TRAP) and tartrate-resistant acid adenosine triphosphatase (TrATPase) in bone, bone marrow and spleen: implications for osteoclast ontogeny [J]. Bone Miner, 1990, 10 (2): 109-19.

18. Kiyoi T. Bone Resorption Activity in Mature Osteoclasts [J]. Methods Mol Biol, 2018, 1868: $215-222$.

19. Holliday LS, Faria LP, Rody WJ Jr. Actin and Actin-Associated Proteins in Extracellular Vesicles Shed by Osteoclasts. Int J Mol Sci. 2019 Dec 25;21(1):158.

20. Qi XC, et al. Protective effect of hyperoside against hydrogen per oxide-induced dysfunction and oxidative stress in osteoblastic MC3T3-E1 cells [J]. Artif Cells Nanomed Biotechnol, 2020, 48 (1): 377-383.

21. Matsubara T, et al. The Actin-Binding Protein PPP1r18 Regulates Maturation, Actin Organization, and Bone Resorption Activity of Osteoclasts. Mol Cell Biol. 2018 Jan 29;38(4):e00425-17.

22. Roscher A, et al. The F-actin modulator SWAP-70 controls podosome patterning in osteoclasts. Bone Rep. 2016 Jul $19 ; 5: 214-221$.

23. Kuroda Y, Matsuo K. Molecular mechanisms of triggering, amplifying and targeting RANK signaling in osteoclasts [J]. World J Orthop, 2012, 3 (11): 167-74

24. Asagiri M, et al. Autoamplification of NFATc1 expression determines its essential role in bone homeostasis [J]. J Exp Med, 2005, 202 (9): 1261- 9. 
25. Matsuo K, et al. Nuclear factor of activated T-cells (NFAT) rescues osteoclastogenesis in precursors lacking c-Fos [J]. J Biol Chem, 2004, 279 (25): 26475-80.

26. Kim K, et al. Inhibitory Effect of Purpurogallin on Osteoclast Differentiation in Vitro through the Downregulation of c-Fos and NFATc1 [J]. Int J Mol Sci, 2018, 19 (2): 601.

27. Oh $\mathrm{JH}$, et al. Insulin enhances RANKL-induced osteoclastogenesis via ERK1/2 activation and induction of NFATc1 and Atp6v0d2 [J]. Cell Signal, 2015, 27 (12): 2325-31.

28. Matsumoto $\mathrm{M}$, et al. Essential role of $\mathrm{p} 38$ mitogen-activated protein kinase in cathepsin $\mathrm{K}$ gene expression during osteoclastogenesis through association of NFATc1 and PU.1 [J]. J Biol Chem, 2004, 279 (44): 45969-79.

29. Hayman AR. Tartrate-resistant acid phosphatase (TRAP) and the osteoclast/immune cell dichotomy [J]. Autoimmunity, 2008, 41 (3): 218-23.

30. Harada K, et al. Polyphosphate-mediated inhibition of tartrate-resistant acid phosphatase and suppression of bone resorption of osteoclasts. PLoS One. 2013 Nov 4;8(11):e78612. 